\title{
Myotubular/centronuclear myopathy and central core disease
}

\author{
Chieko Fujimura-Kiyono, Gabor Z. Racz, Ichizo Nishino \\ Department of Neuromuscular Research, National Institute of Neuroscience, National Center of Neurology and Psychiatry, 4-1-1 \\ Ogawahigashi-Cho Kodaira, Tokyo 187-8502, Japan
}

\begin{abstract}
The term congenital myopathy is applied to muscle disorders presenting with generalized muscle weakness and hypotonia from early infancy with delayed developmental milestones. The congenital myopathies have been classified into various categories based on morphological findings on muscle biopsy. Although the clinical symptoms may seem homogenous, the genetic basis is remarkably variable. This review will focus on myotubular myopathy, centronuclear myopathy, central core disease, and congenital neuromuscular disease with uniform Type 1 fiber, myopathies that are subjects of our ongoing examinations.
\end{abstract}

Key words: Central core disease, centronuclear myopathy, congenital myopathy, congenital neuromuscular disease with uniform Type 1 fibers, myotubular myopathy

\section{Introduction}

Congenital myopathies collectively denote a term used to describe a heterogeneous group of inherited muscle diseases that are typically present at birth or in the first year of life with reduced fetal movements or delay of motor milestones, as well as weakness and hypotonia. The diagnosis of a specific congenital myopathy relies predominantly on muscle pathology that reveals distinctive changes within the myofiber under light and/or electron microscopy, and not in the sarcolemmal membrane, which is the origin of the muscular dystrophies.

Epidemiological data are available for congenital myopathies as a general group but not for specific diseases or conditions. The incidence of all congenital myopathies is estimated at around 6.0/100.000 live births, or one-tenth of all cases of neuromuscular disorders. ${ }^{[1]}$ In our series from 1979-2007, we had 597 cases as congenital myopathy among 10332 muscle biopsies diagnosed based on muscle pathology findings.
Despite their genetic heterogeneity, the clinical features have some characteristics in common. Main symptoms are generalized muscle weakness and hypotonia, typically presenting at birth or in infancy. The clinical severity is highly variable from a mild, almost asymptomatic form, to a severe form characterized by marked hypotonia at birth. Muscle weakness can be very subtle in childhood and thus can only present during adult years. Dysmorphic facies is very common due to facial muscle involvement, and when this does occur, the onset is thought to be in utero. High arched palate is often documented in almost all patients with this disease. Scoliosis and contracture of joints are seen early in childhood, especially in infantile or child onset form. On the other hand, cardiac involvement is rare and intelligence is usually normal. Clinically, it is sometimes difficult to distinguish a congenital myopathy from spinal muscular atrophy or congenital myotonic dystrophy solely based upon clinical features. Serum creatine kinase levels are within the normal range or are mildly elevated at most.

Pathologically, Type 1 fiber predominance and Type 1 fiber hypotrophy are commonly seen features. In addition, the presence of characteristic structural abnormalities such as nemaline bodies, central cores and central nuclei, are the diagnostic hallmark of subtypes of congenital myopathies.

Their clinical course is usually non-progressive or slowly progressive. Prognosis is often determined by respiratory, orthopedic, and bulbar involvement.

Over the past decade, a number of causative genes have been identified for congenital myopathies. But up to this time, only a few of these genes have been well characterized and have clarified the pathomechanism underlying the disease.

\section{Centronuclear Myopathy}

Centronuclear myopathy (CNM) comprises a group of 
congenital myopathies which are defined pathologically by the presence of the characteristic centrally located nuclei in a large number of muscle fibers. Although the pathological findings are uniform, the clinical features and genetic background are heterogeneous. CNMs can be classified into three forms: X-linked (OMIM 310400), autosomal recessive (OMIM 255200) and autosomal dominant (OMIM 160150) forms. ${ }^{[2,3]}$ CNM was first reported under the name of "myotubular myopathy" by Spiro et al., in 1966. ${ }^{[4]}$ The term "myotubular myopathy" was used due to morphological similarities to fetal myotubes. Currently, however, the use of this term is limited to indicate only the severe infantile form, which is usually X-linked.

Unlike in other congenital myopathies, ptosis and extraocular muscle involvement are frequently seen in CNMs. In addition, facial muscle involvement can be prominent, resulting in the characteristic "elongated" facies. Central or peripheral nervous systems are occasionally affected..$^{[5]}$

\section{X-linked Myotubular Myopathy}

X-linked myotubular myopathy (XLMTM) is caused by mutations in MTM1 that encodes myotubularin and is transmitted in X-linked recessive mode. The estimated incidence is approximately one out of 50,000 males. ${ }^{[6]}$ Myotubularin is a protein-tyrosine phosphatase required for muscle cell differentiation, and has been demonstrated to be a phosphatidylinositol 3-phosphate phosphatase. It is highly conserved during evolution and plays important roles in regulating intracellular membrane trafficking and vesicular transport processes. ${ }^{[7,8]}$

Myonuclear position is known to change with developmental stage. In fetal muscle, the nuclei are positioned at the center of muscle fibers, however, the majority of the nuclei are found at the periphery at birth. These pathological features resemble those of fetal muscles or myotubes, therefore XLMTM had been attributed to an arrest of myogenesis. ${ }^{[5]}$ However, muscle development and differentiation were normal in MTM1 knockout mice and a myopathy with centralized nuclei was seen only after four weeks of age, clearly indicating that centrally-placed nuclei are not due to the arrest of myogenesis and that myotubularin plays a role in skeletal muscle maintenance rather than myogenesis. ${ }^{[9]}$

To date, more than 200 different mutations have been identified. Originally, MTM1 mutations were associated with the severe infantile form but now we know that MTM1 mutations can also cause milder phenotypes. In the study of genotype-phenotype correlation, only 17 and seven individuals of the 116 cases were classified as mild and intermediate phenotype. ${ }^{[10]}$ Furthermore, missense mutation in MTM1 was identified in a 67-year-old man, who was initially suspected to have autosomal centronuclear myopathy, and whose two grandsons also show mild phenotype. ${ }^{[11]}$ Truncating mutations usually cause the severe neonatal form while non-truncating and missense mutations can lead to either mild or severe phenotype..$^{[10,11]}$

\section{Clinical characteristics}

Typically, patients with XLMTM are born with severe generalized muscle hypotonia and weakness. Respiratory insufficiency is frequently seen at birth so patients require ventilatory support. Previously, most patients died due to respiratory insufficiency. [2] Recently, however, their length of survival has been significantly prolonged due to the advancement of respiratory care. Many patients also need tube feeding due to difficulty in sucking or swallowing. Ptosis, extraocular muscle weakness and facial muscle involvement are frequently seen. Prenatal history often shows polyhydramnios and fetal hypokinesia, indicating the disease has started in utero. Patients' mothers sometimes have a history of miscarriages and stillbirths. The majority of heterozygous females are asymptomatic. Nevertheless, muscle weakness can be seen in rare cases due to skewed or random $\mathrm{X}$-chromosome inactivation. ${ }^{[12,13]}$ Generally, their symptoms are less severe in females compared to affected males.

A wide variety of medical complications, such as vitamin $\mathrm{K}$-dependent coagulopathy, gastrointestinal bleeding, pyloric stenosis, cholestatis/cholelithiasis, scoliosis and nephrocalcinosis/kidney stones, are seen in XLMTM and can sometimes be life-threatening. Therefore, clinicians should carefully monitor patients for these potential complications. ${ }^{[14]}$

\section{Pathology}

Muscle pathology in typical infantile cases is characterized by the presence of numerous small and round myofibers with centrally placed nuclei and peripheral halo [Figure 1], a clear zone with reduced oxidative enzyme activity in the periphery of muscle fibers. The percentage of fibers with centrally placed nuclei is widely variable and its proportion does not correlate with disease severity. ${ }^{[15]}$ Necrotic and regenerating fibers, fibrosis, and inflammatory cell infiltration are usually not seen. Type 1 predominance is often seen as in other congenital myopathies. Both Type 1 and 2 fibers are hypotrophic. However, Type 1 fibers are more remarkably affected. ${ }^{[16]}$

Prognosis has been correlated with pathologic findings. Patients with larger myofibers tend to have better outcome and frequently become independent from respirator. In contrast, patients with smallcalibered fibers continue to be ventilator-dependent and have poor prognosis. ${ }^{[16]}$ 


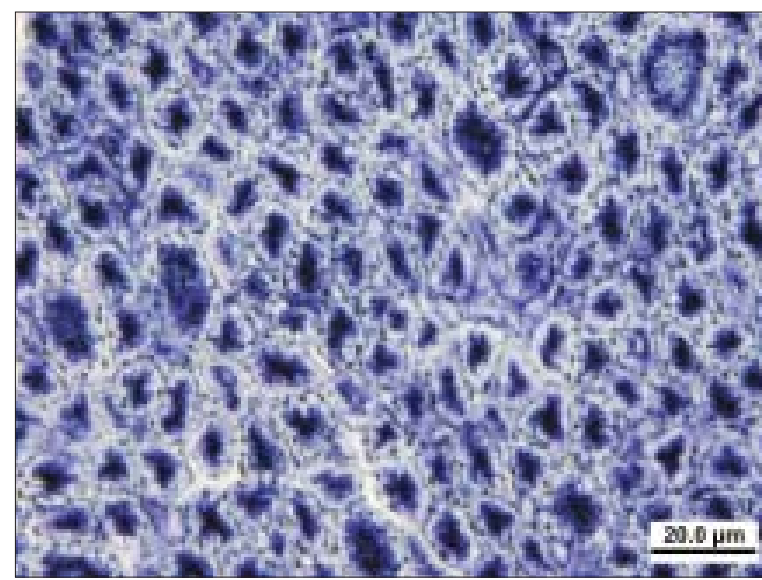

Figure 1: Myotubular myopathy, NADH-TR staining. Note the central nuclei surrounded by rim of densely stained material and the peripheral halo.

The abovementioned pathological findings are very similar to those in congenital myotonic dystrophy. In fact, it is also clinically difficult to differentiate between XLMTM and congenital myotonic dystrophy, as both conditions present with severe hypotonia and muscle weakness at birth. Furthermore, recently, a newborn with spinal muscular atrophy (SMA) Type 0 showing similar clinicopathological findings with myotubular myopathy was reported. ${ }^{[17]}$ Therefore, not only congenital myotonic dystrophy but also SMA Type 0 should be included in the differential diagnosis.

\section{Autosomal Dominant and Recessive Centronuclear Myopathy}

Autosomal dominant and recessive forms of CNM (AD-CNM, AR-CNM) have much more variable clinical characteristics, but have later onset and milder clinical course in most cases. ${ }^{[2]}$

Mutations in DNM2 are responsible for autosomal dominant AD-CNM. ${ }^{[18]}$ DNM2 gene encodes dynamin 2, a large guanosin triphophatase (GTPase) that plays roles in endocytosis, membrane trafficking, actin assembly and centrosome cohesion. ${ }^{[19-22]}$ Dynamin 2 has five domains: 1) N-terminal tripartite GTPase, 2) middle,3) pleckstrin homology, 4) GTPase effector, and 5) C-terminal proline rich domains. So far all reported DNM2 mutations associated with typical late-onset $\mathrm{AD}-\mathrm{CNM}$ are located in the middle domain. In contrast, mutations associated with rare severe infantile cases are present in the pleckstrin homology domain. ${ }^{[23]}$ DNM2 mutations also cause Charcot-MarieTooth disease Type 2B (CMT2B), for which mutations in the pleckstrin homology domain have been reported. ${ }^{[24,25]}$ The pleckstrin homology domain can function as regulated membrane-binding module that binds to inositol lipids and responds to upstream signals by targeting the host proteins to the correct cellular sites.

Recently, two new genes have been added to the list: hJUMPY and BIN1. Two missense mutations in hJUMPY were identified initially in sporadic cases of CNM. ${ }^{[26]}$ hJUMPY is a phosphoinositide phosphatase that shares the same substrate specificity as myotubularin. However, its pathomechanism and mode of inheritance are still unknown. BIN1 encodes amphiphysin 2 that plays a role in membrane remodeling and tubulation by interacting with dynamin 2. ${ }^{[27]}$ Probably, both amphiphysin 2 and dynamin 2 play a role in normal positioning of myonuclei. More recently, a sporadic CNM patient with a de novo dominant missense mutation in the ryanodine receptor (RYR1) was reported. In this patient, quadriceps femoris muscle biopsied at age one year showed muscle pathology of CNM; however, core-like structure was seen in tibialis anterior muscle biopsied at age nine years. ${ }^{[28]}$ Probably, more cases are necessary to conclude that RYR1 is the causative gene for CNM.

\section{Clinical characteristics}

The age of onset is variable among AD-CNM, ARCNM and sporadic CNM. However, disease severity in AD-CNM tends to be milder as compared with AR-CNM and sporadic CNM. ${ }^{[29]}$ For example, in DNM2-related AD-CNM (DNM2-CNM), the onset of the disease is after age 12 years and it can be as late as 74 years. ${ }^{[30]}$

Muscle weakness is seen with variable severity. Limb girdle and paraspinal muscles have been thought to be the most severely affected muscles in several CNM patients. However, recently, a study showed that the distribution of muscle weakness is actually more variable. ${ }^{[29]}$ In fact, in DNM2-CNM, distal limb muscles, especially calf muscles and posterior thigh muscles, are more preferentially affected than proximal limb and axial muscles. ${ }^{[30]}$ Achilles tendon contractures are also frequently observed. Ptosis is commonly seen in patients, as in XLMTM, but extraocular muscle weakness is only occasionally seen. ${ }^{[30]}$ The central nervous system is occasionally affected and mild cognitive impairment can be seen. ${ }^{[4,30]}$

Neuropathic signs, such as absence of tendon reflexes on neurological examination and fibrillations or reduction of the compound muscle action potential (CMAP) on electrophysiological examination, are sometimes seen in DNM2-CNM patents. This suggests that there may be overlapping features between DNM2CNM and DNM2-CMT2B phenotypes.

\section{Pathology}

Fiber size of CNM has much more variation than XLMTM; in addition, fibers generally appear less rounded and more polygonal compared with XLMTM. This is because CNM has milder phenotype and biopsies 
are performed at older ages than XLMTM. Centrally placed nuclei are seen not only in small fibers but also in normal or hypertrophic fibers. In longitudinal sections, these nuclei occasionally form chain. More than $80 \%$ of myofibers have centrally placed nuclei in $50 \%$ of AR-CNM and $40 \%$ of AD-CNM cases. ${ }^{[29]}$ Typically, in DNM2-CNM, radially-arranged sarcoplasmic strands are seen on nicotinamide adenosine dinucleotidetetrazolium reductase (NADH-TR) staining [Figure 2]. Type 1 fiber predominance and hypotrophy are often seen, but are thought to be nonspecific as these can be seen in other conditions. ${ }^{[29]}$

\section{Central Core Disease}

Central core disease (CCD; OMIM 117000) is characterized by clinical features of a congenital myopathy and a particular histological picture with areas of reduced oxidative activity in Type 1 (slow) muscle fibers, the "central cores" [Figure 3]. CCD was originally reported in a family with congenital

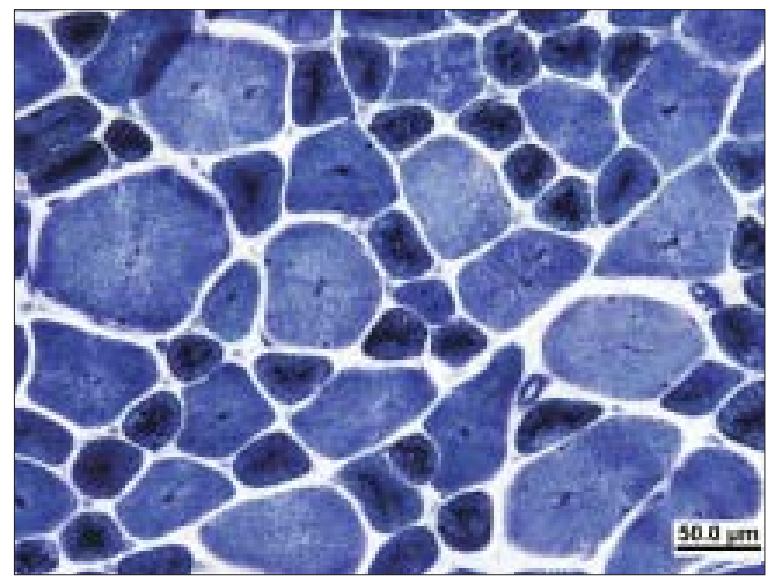

Figure 2: Autosomal dominant centronuclear myopathy, with dynamin 2 mutation, NADH-TR staining. Type 1 fiber atrophy, central nuclei and radial sarcoplasmic strands are seen.

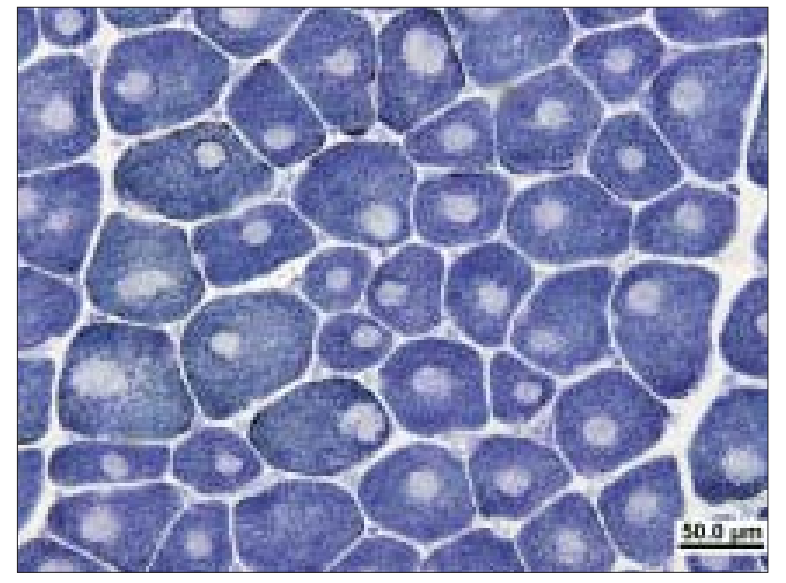

Figure 3: Histopathology of central core disease: NADH-TR, transverse section. Predominance of darkly stained, Type 1 fibers with well-demarcated cores in virtually all fibers. hypotonia, non-progressive and non-wasting weakness of the proximal muscles of the limbs, more than half a century ago. ${ }^{[31]}$ The term "central core" was introduced to indicate the area with characteristic absence of oxidative enzyme activity in the muscle fiber on NADHTR staining, ${ }^{[32,33]}$ the absence of mitochondria can be confirmed by electron microscopy. ${ }^{[34]}$

\section{Genetic background}

CCD is associated with mutations in the skeletal muscle RYR1 gene at Chromosome 19q13.1. RYR1 is one of the largest human genes with 106 exons encoding RYR1 protein, which consists of 5037 amino acids and is expressed mainly in skeletal muscles. ${ }^{[35]}$ The functional ryanodine receptor is a homotetramer of single RYR1 units and it is ligand-gated release channel for $\mathrm{Ca}^{2+}$ stored in the terminal cisterna with a crucial role in excitation-contraction (E-C) coupling. The actual calcium release channel is located in the C-terminal part of the RYR1 protein, while the $\mathrm{N}$-terminal part is sarcoplasmic and constitutes the electron-microscopically visible foot structure that interacts with the dihydropyridine receptor (DHPR) and other regulating proteins. ${ }^{[36]}$ Calcium release is primarily triggered by voltage-induced conformational changes of the DHPR, and secondarily by a number of exogenous and endogenous effector molecules and associated proteins.

Altogether, more than 80 point mutations have been identified in the RYR1 gene. Most mutations are missense but a few small deletions and cryptic splicing site mutations have also been documented. ${ }^{[37]}$ Most cases of RYR1-associated diseases are autosomal dominant; however, rare cases are autosomal recessive. Mutations in RYR1 are also associated with malignant hyperthermia susceptibility (MHS), multi-minicore disease $(\mathrm{MmD})$, and CNM, although rarely. ${ }^{[38]}$ The association between CCD and MHS had been suspected early, as individuals with MHS may have central cores on muscle biopsy ${ }^{[39]}$ and patients with CCD tend to have malignant hyperthermia episodes during general anesthesia induced by halogenated anesthetic agents and succinylcholine. However, MHS patients usually do not have any myopathy before developing $\mathrm{MH}$ syndrome. In our series, 50\% of MHS cases with RYR1 mutations had cores on muscle pathology; however, none of them were typical, well-demarcated, "central cores", suggesting that those with typical CCD may not have MHS although further studies are necessary to draw any conclusion.

In an extensive screening of all 106 exons in 27 Japanese CCD patients, mutations were identified in $25(93 \%) .{ }^{[40]}$ In fact, two cases without mutations had unusual cores, suggesting that virtually all CCD are due to RYR1 mutations. Before this report, RYR1 mutation screening for CCD cases had been limited 
to three "hotspots", with particular attention to the C-terminal-encoding exons; therefore only about $60 \%$ of CCD mutations had been assigned to RYR1, and the remaining were unidentified. In our series, most mutations were located in Exon 101-102, but novel mutations were found in Exon 47-48, as well as one in Exon 13.

Recessive RYR1 mutations are much less frequent, and distributed along the entire gene, causing atypical CCD and MmD. ${ }^{[41]}$ Zhou et al., discovered that in half of their patients with recessive core myopathies epigenetic tissue-specific allelic silencing (leading to monoallelic transcription) unveils recessive RYR1 mutation in skeletal muscle, despite being heterozygous at the genomic level. ${ }^{[42]}$ These data also suggest that imprinting by methylation is a likely mechanism for this phenomenon, and similar mechanisms can play a general role in human phenotypic heterogeneity as well as in irregularities of inheritance patterns. In addition, compound heterozygosity for RYR1 null and missense mutation has been recently identified as another important mechanism of monoallelic RYR1 expression. ${ }^{[43]}$

How the mutations affect the channel function in CCD, is still controversial. One hypothesis is that these mutations lead to leaky channels, depletion of $\mathrm{SR} \mathrm{Ca}^{2+}$ stores and consequently muscle weakness ${ }^{[44]}$ together with compensatory uptake of the excess $\mathrm{Ca}^{2+}$ by mitochondria, which may lead to mitochondrial dysfunction and loss. ${ }^{[45]}$ Alternatively, C-terminal RYR1 mutations lead to functional uncoupling of sarcolemma depolarization from release of $\mathrm{Ca}^{2+}$ from the SR calcium stores with consequential muscle weakness. ${ }^{[46]}$

Rare non-RYR1 mutations include $\beta$-myosin heavy chain mutation that cause CCD in familial hypertrophic cardiomyopathy. ${ }^{[47}$

\section{Clinicopathologic features}

The clinical features are quite variable, ranging from the absence of symptoms to severe phenotype as manifested by a lack of independent ambulation. ${ }^{[48]}$ This clinical and pathological variability applies also to patients within a family. Dominantly inherited CCD starts in infancy with hypotonia or in early childhood with motor developmental delay. Almost all children with CCD achieve the ability to walk independently, except for some of those with congenital hip dislocation and the less frequent recessive neonatal form of CCD that presents as fetal akinesia syndrome. ${ }^{[41]}$ Dominantly inherited CCD is usually non-progressive and respiratory failure is uncommon. Affected patients tend to use the Gowers' maneuver when they stand up. Distribution of weakness is typically proximal with prominent involvement of the hip girdle and also axial muscles although focal muscle wasting has also been reported in a few cases. ${ }^{[49]}$ The most common orthopedic complications are congenital dislocation or subluxation of the hips ${ }^{[50-52]}$ and (kypho)scoliosis, ${ }^{[53]}$ as well as foot deformities including pes equinovarus and pes planus. Many patients have marked hypermobility of the joints, ligamentous laxity, occasionally associated with patellar instability, whereas contractures are rare. ${ }^{[50]}$

Serum creatine kinase (CK) levels are usually normal or only mildly elevated. However, in rare cases, it can be elevated to 6-14 times normal. ${ }^{[54]}$ Muscle ultrasound often shows an increase in echogenicity even in mildly affected cases. A muscle magnetic resonance (MR) imaging study on RYR1-related myopathies showed a consistent pattern characterized by selective involvement of the following thigh and lower leg muscles: vasti, sartorius, adductor magnus and soleus, gastrocnemius and peroneal group. ${ }^{[55]}$ An earlier case report of two Japanese siblings confirmed this with the addition of the involvement of paravertebral muscles. ${ }^{[56]}$ In vitro contracture test or calcium-induced calcium release test confirms the presence of MHS, and thus is recommended in CCD patients before general anesthesia. ${ }^{[52,57]}$

On muscle pathology, the core structure is most clearly demonstrated with oxidative enzyme stains such as NADH-TR, succinate dehydrogenase, and cytochrome c oxidase, because mitochondria and sarcoplasmic reticulum are absent in this area. The cores typically extend along the muscle fibers. Periodic acid-Schiff stains the central part more intensely than the periphery of the fiber.

With immunostaining, in the cores myofibrillar proteins ( $\alpha \mathrm{B}$-crystallin, desmin, filamin $\mathrm{C}$; these are nonspecific, seen also in target fibers in various denervating diseases), and calcium-related proteins (SERCA, triadin) can be detected, whereas RYR1 is depleted. ${ }^{[58]}$ Nemaline rods can be detected in a minority of CCD cases. ${ }^{[59]}$ Electron-microscopic features demonstrated the extensive disorganization of sarcomere in the cores, shown by a severe fragmentation and decrease of Z-bands. ${ }^{[60,61]}$

Interestingly, there is clear genotype-phenotype correlation in CCD. Mutations in the C-terminal region of RYR1 are associated with typical CCD features: characteristic, well-demarcated cores are seen, in addition to the near-absence of Type 2 fibers (in other words, almost all fibers are Type 1). In contrast, patients with mutations in the non-C-terminal region have atypical cores that are characterized with vague margin and are often multiple and peripherally-located in the muscle fiber. ${ }^{[40]}$ In these patients, Type 2 fibers are variably present.

There is no association between the number of cores on muscle biopsy and the degree of muscle weakness. Those with C-terminal RYR1 mutations usually have more severe symptoms with muscle weakness and scoliosis while those with non-C-terminal mutations 
rarely have muscle weakness although all patients with non-C-terminal mutations so far examined had MHS in our series. ${ }^{[40]}$

\section{Congenital Neuromuscular Disease with Uniform Type 1 Fibers}

Congenital neuromuscular disease with uniform Type 1 fibers (CNMDU1) is defined as a congenital myopathy characterized by the almost exclusive presence of Type 1 muscle fiber (>99\%) without any other striking pathological changes. It was first described in 1983, ${ }^{[62]}$ since then, 12 reports have been published. In our laboratory, we have 17 documented cases of CNMDU1.

As discussed above, CCD due to C-terminal mutations is accompanied by the absence of Type 2 fibers [Figure 4], which is uniform Type 1 fiber, in other words, regardless of the presence of central cores, making RYR1 a potential causative gene. Indeed, sequencing of the RYR1 gene in cases with CNMDU1 revealed C-terminal mutations in four of 10 patients. ${ }^{[63]}$ In fact, the clinical manifestations of the four CNMDU1 patients with C-terminal mutations are similar to those of the CCD cases due to the C-terminal mutations, regardless of the presence or absence of cores on muscle pathology, raising a possibility that they are actually the same disease, and that the absence of cores may be attributed to muscle sampling, as the degree of affectation could be different among muscles. Alternatively, cores may be formed in an age-dependent manner and in CNMDU1 cases, muscle was biopsied before core was formed. Nevertheless, in the other six cases more severe symptoms were commonly seen, such as poor fetal movement, respiratory distress and mental retardation, suggesting the involvement of the nervous system as well. Such a rare case was first reported in $1991^{[64]}$ but to date the causative gene(s) has not been discovered.

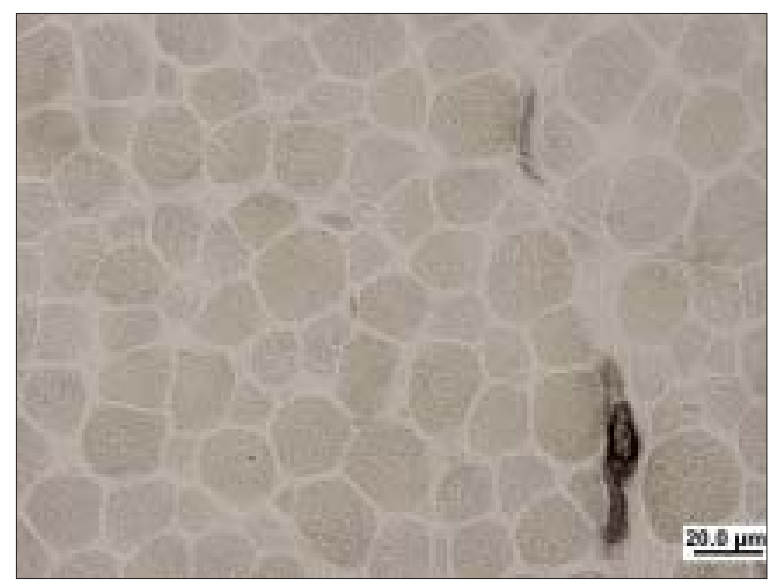

Figure 4: Histopathology of CNDMU1, myosin ATPase staining at pH 10.4 (stains Type 2, fast fibers). No fiber contains fast myosin heavy chain isoform. Besides variation in fiber size, marked fibrosis can be observed.

\section{Treatment and Perspectives}

No curative therapy is currently available for congenital myopathies. The management is essentially supportive and/or rehabilitative, based on a multidisciplinary approach. Physiotherapy is utilized for the maximum preservation of muscle power and function and the prevention of contractures, with particular care after surgical procedures. $\beta$-agonists have been shown to be beneficial on muscle strength in healthy individuals ${ }^{[65]}$ and also in CCD and MmD patients. ${ }^{[66]}$ This may be due to the $\beta$-adrenergic mediated fast-to-slow fiber type conversion with subsequent hypertrophy and/ or reduction in wasting, however this applies only for non-progressive diseases. ${ }^{[67]}$

Recent advances in identifying causative genes of the congenital myopathies, better understanding the pathomechanism, and rapidly developing diagnostic methods can provide prenatal diagnosis for these entities, although often only on a research basis. Ultimately, rational molecular therapies may be invented in the future, as most of the muscle diseases are still awaiting specific and effective treatment.

\section{References}

1. Wallgren-Pettersson C: Congenital nemaline myopathy: A longitudinal study. In Commentationes Physico Mathematicae University of Helsinki; 1990.

2. Wallgren-Pettersson C, Clarke A, Samson F, Fardeau M, Dubowitz V, Moser H, \& al. The myotubular myopathies: Differential diagnosis of the $\mathrm{X}$ linked recessive, autosomal dominant, and autosomal recessive forms and present state of DNA studies. J Med Genet 1995;32:673-9.

3. Pierson CR, Tomczak K, Agrawal P, Moghadaszadeh B, Beggs AH. X-linked myotubular and centronuclear myopathies. J Neuropathol Exp Neurol 2005;64:555-64

4. Spiro AJ, Shy GM, Gonatas NK. Myotubular myopathy: Persistence of fetal muscle in an adolescent boy. Arch Neurol 1966;14:1-14.

5. Echaniz-Laguna A, Nicot AS, Carré S, Franques J, Tranchant C, Dondaine N, et al. Subtle central and peripheral nervous system abnormalities in a family with centronuclear myopathy and a novel dynamin 2 gene mutation. Neuromuscul Disord 2007;17:955-9.

6. Laporte J, Biancalana V, Tanner SM, Kress W, Schneider V, WallgrenPettersson C, etal. MTM1 mutations in X-linked myotubular myopathy. Hum Mutat 2000;15:393-409.

7. Taylor GS, Maehama T, Dixon JE. Inaugural article: myotubularin, a protein tyrosine phosphatase mutated in myotubular myopathy, dephosphorylates the lipid second messenger, phosphatidylinositol 3-phosphate. Proc Natl Acad Sci USA 2000;97:8910-5.

8. Blondeau F, Laporte J, Bodin S, Superti-Furga G, Payrastre B, Mandel JL. Myotubularin, a phosphatase deficient in myotubular myopathy, acts on phosphatidylinositol 3-kinase and phosphatidylinositol 3-phosphate pathway. Hum Mol Genet 2000;9:2223-9.

9. Buj-Bello A, Laugel V, Messaddeq N, Zahreddine H, Laporte J, Pellissier JF, \& al. The lipid phosphatase myotubularin is essential for skeletal muscle maintenance but not for myogenesis in mice. Proc Natl Acad Sci USA 2002;99:15060-5.

10. McEntagart M, Parsons G, Buj-Bello A, Biancalana V, Fenton I, Little M, \&al. Genotype-phenotype correlations in X-linked myotubular myopathy. Neuromuscul Disord 2002;12:939-46.

11. Biancalana V, Caron O, Gallati S, Baas F, Kress W, Novelli G, \& al. Characterization of mutations in 77 patients with X-linked myotubular myopathy, including a family with a very mild phenotype. Hum Genet 
2003;112:135-42.

12. Tanner SM, Orstavik KH, Kristiansen M, Lev D, Lerman-Sagie T, Sadeh $\mathrm{M}$, $\mathrm{A}$ al. Skewed X-inactivation in a manifesting carrier of X-linked myotubular myopathy and in her non-manifesting carrier mother. Hum Genet 1999;104:249-53.

13. Hammans SR, Robinson DO, Moutou C, Kennedy CR, Dennis NR, Hughes PJ, \& al. A clinical and genetic study of a manifesting heterozygote with X-linked myotubular myopathy. Neuromuscul Disord 2000;10:133-7.

14. Herman GE, Finegold M, Zhao W, de Gouyon B, Metzenberg A. Medical complications in long-term survivors with X-linked myotubular myopathy. J Pediatr 1999;134:206-14.

15. Helliwell TR, Ellis IH, Appleton RE. Myotubular myopathy: Morphological, immunohistochemical and clinical variation. Neuromuscul Disord 1998;8:152-61.

16. Pierson CR, Agrawal PB, Blasko J, Beggs AH. Myofiber size correlates with MTM1 mutation type and outcome in X-linked myotubular myopathy. Neuromuscul Disord 2007;17:562-8.

17. Nadeau A, D'Anjou G, Debray G, Robitaille Y, Simard LR, Vanasse M. A newborn with spinal muscular atrophy type 0 presenting with a clinicopathological picture suggestive of myotubular myopathy. J Child Neurol 2007;22:1301-4.

18. Bitoun M, Maugenre S, Jeannet PY, Lacène E, Ferrer X, Laforêt P, đ al. Mutations in dynamin 2 cause dominant centronuclear myopathy. Nat Genet 2005;37:1207-9.

19. Jones SM, Howell KE, Henley JR, Cao H, MeNiven MA. Role of dynamin in the formation of transport vesicles from the trans-Golgi network. Science 1998;279:573-7.

20. Praefcke GJ, McMahon HT. The dynamin superfamily: Universal membrane tubulation and fission molecules? Nat Rev Mol Cell Biol 2004;5:133-47.

21. Orth JD, MeNiven MA. Dynamin at the actin-membrane interface. Curr Opin Cell Biol 2003;15:31-9.

22. Thompson HM, Cao H, Chen J, Euteneuer U, MeNiven MA. Dynamin 2 binds gamma-tubulin and participates in centrosome cohesion. Nat Cell Biol 2004;6:335-42.

23. Bitoun M, Bevilacqua JA, Prudhon B, Maugenre S, Taratuto AL, Monges $\mathrm{S}, \mathbb{e}$ al. Dynamin 2 mutations cause sporadic centronuclear myopathy with neonatal onset. Ann Neurol 2007;62:666-70.

24. Züchner S, Noureddine M, Kennerson M, Verhoeven K, Claeys K, De Jonghe $\mathrm{P}, \mathbb{\notin}$ al. Mutations in the pleckstrin homology domain of dynamin 2 cause dominant intermediate Charcot-Marie-Tooth disease. Nat Genet 2005;37:289-94.

25. Fabrizi GM, Ferrarini M, Cavallaro T, Cabrini I, Cerini R, Bertolasi L, \& al. Two novel mutations in dynamin-2 cause axonal Charcot-Marie-Tooth disease. Neurology 2007;69:291-5.

26. Tosch V, Rohde HM, Tronchère H, Zanoteli E, Monroy N, Kretz C, đal. A novel PtdIns3P and PtdIns(3,5)P2 phosphatase with an inactivating variant in centronuclear myopathy. Mol Genet 2006;15:3098-106.

27. Nicot AS, Toussaint A, Tosch V, Kretz C, Wallgren-Pettersson C, Iwarsson $\mathrm{E}, \mathbb{A}$ al. Mutations in amphiphysin 2 (BIN1) disrupt interaction with dynamin 2 and cause autosomal recessive centronuclear myopathy. Nat Genet 2007;39:1134-9.

28. Jungbluth H, Zhou H, Sewry CA, Robb S, Treves S, Bitoun M, \& al. Centronuclear myopathy due to a de novo dominant mutation in the skeletal muscle ryanodine receptor (RYR1) gene. Neuromuscul Disord 2007;17:338-45.

29. Jeannet PY, Bassez G, Eymard B, Laforêt P, Urtizberea JA, Rouche A, \& al. Clinical and histologic findings in autosomal centronuclear myopathy. Neurology 2004;62:1484-90.

30. Fischer D, Herasse M, Bitoun M, Barragán-Campos HM, Chiras J, Laforêt $\mathrm{P}$, $\mathrm{A}$ al. Characterization of the muscle involvement in dynamin 2-related centronuclear myopathy. Brain 2006;129:1463-9.

31. Magee KR, Shy GM. A new congenital non-progressive myopathy. Brain 1956;79:610-21.

32. Greenfield JG, Cornman T, Shy GM. The prognostic value of the muscle biopsy in the floppy infant. Brain 1958;81:461-84.

33. Dubowitz V, Pearse AG. Oxidative enzymes and phosphorylase in centralcore disease of muscle. Lancet 1960;2:23-4.

34. Seitelberger F, Wanko T, Gavin MA The muscle fiber in central-core disease. Histochemical and electronmicroscopic observations. Acta Neuropath. (Berlin) 1962;1:223-37.

35. Phillips MS, Fujii J, Khanna VK, DeLeon S, Yokobata K, de Jong PJ, \& al. The structural organization of the human skeletal muscle ryanodine receptor (RYR1) gene. Genomics 1996;34:24-41.

36. Wagenknecht T, Grassucci R, Frank J, Saito A, Inui M, Fleischer S. Three-dimensional architecture of the calcium channel/foot structure of sarcoplasmic reticulum. Nature 1989;338:167-70.

37. Robinson R, Carpenter D, Shaw MA, Halsall J, Hopkins P. Mutations in RYR1 in malignant hyperthermia and central core disease. Hum Mutat $2006 ; 27: 977-89$

38. Jungbluth H, Zhou H, Sewry CA, Robb S, Treves S, Bitoun M, \& al. Centronuclear myopathy due to a de novo dominant mutation in the skeletal muscle rvanodine receptor (RYR1) gene. Neuromuscul Disord $2007 ; 17: 338-345$.

39. Denborough MA, Dennett X, Anderson RM. Central-core disease and malignant hyperpyrexia. Br Med J 1973;1:272-3.

40. Wu S, Ibarra MC, Malicdan MC, Murayama K, Ichihara Y, Kikuchi H, \& al. Central core disease is due to RYR1 mutations in more than $90 \%$ of patients. Brain 2006;129:1470-80.

41. Romero NB, Monnier N, Viollet L, Cortey A, Chevallay M, Leroy JP, \& al. Dominant and recessive central core disease associated with RYR1 mutations and fetal akinesia. Brain 2003;126:2341-9.

42. Zhou H, Brockington M, Jungbluth H, Monk D, Stanier P, Sewry CA, đ al. Epigenetic allele silencing unveils recessive RYR1 mutations in core myopathies. Am J Hum Genet 2006;79:859-68.

43. Monnier N, Marty I, Faure J, Castiqlioni C, Desnuelle C, Sacconi S, \& al. Null mutations causing depletion of the type 1 ryanodine receptor (RYR1) are commonly associated with recessive structural congenital myopathies with cores. Hum Mutat 2008;29:670-8.

44. Lynch PJ, Tong J, Lehane M, Mallet A, Giblin L, Heffron JJ, \& al A mutation in the transmembrane/luminal domain of the ryanodine receptor is associated with abnormal Ca2 + release channel function and severe central core disease. Proc Natl Acad Sci USA 1999;96:4164-9.

45. Bernardi P, Rasola A. Calcium and cell death: The mitochondrial connection. Subcell Biochem 2007;45:481-506.

46. Rossi AE, Dirksen RT. Sarcoplasmic reticulum: The dynamic calcium governor of muscle. Muscle Nerve 2006;33:715-31.

47. Fananapazir L, Dalakas MC, Cyran F, Cohn G, Epstein ND. Missense mutations in the beta-myosin heavy-chain gene cause central core disease in hypertrophic cardiomyopathy. Proc Natl Acad Sci USA 1993;90:3993-7.

48. Quinlivan RM, Muller CR, Davis M, Laing NG, Evans GA, Dwyer J, et al. Central core disease: Clinical, pathological, and genetic features. Arch Dis Child 2003;88:1051-5

49. Dubowitz V, Platts M. Central core disease of muscle with focal wasting. J Neurol Neurosurg Psychiatry 1965;28:432-7.

50. Gamble JG, Rinsky LA, Lee JH. Orthopaedic aspects of central core disease. J Bone Joint Surg Am 1988;70:1061-6.

51. Ramsey PL, Hensinger RN. Congenital dislocation of the hip associated with central core disease. J Bone Joint Surg Am 1975;57:648-51.

52. Eng GD, Epstein BS, Engel WK, McKay DW, McKay R. Malignant hyperthermia and central core disease in a child with congenital dislocating hips. Arch Neurol 1978;35:189-97.

53. Sestero AM, Perra JH. A case report of severe kvphoscoliosis and autofusion of the posterior elements in two siblings with central core disease. Spine 2005;30:E50-5.

54. Isaacs H, Heffron JJ, Badenhorst M. Central core disease: A correlated genetic, histochemical, ultramicroscopic, and biochemical study. J Neurol Neurosurg Psychiatry 1975;38:1177-86.

55. Jungbluth H, Davis MR, Müller C, Counsell S, Allsop J, Chattopadhyay A, đal. Magnetic resonance imaging of muscle in congenital myopathies associated with RYR1 mutations. Neuromuscul Disord 2004;14: 785-90.

56. Arai Y, Sumida S, Osawa M, Hirasawa K, Okada N, Kawai M, \& al. Skeletal muscle CT scan and ultrasound imaging in two siblings with central core disease. No To Hattatsu 1990;22:55-60.

57. Robinson RL, Brooks C, Brown SL, Ellis FR, Halsall PJ, Quinnell R.J, \& al. RYR1 mutations causing central core disease are associated with more severe malignant hyperthermia in vitro contracture test phenotypes. 
Hum Mutat 2002;20:88-97.

58. Sewry CA, Müller C, Davis M, Dwyer JS, Dove J, Evans G, \& al. The spectrum of pathology in central core disease. Neuromuscul Disord 2002;12:930-8.

59. Scacheri PC, Hoffman EP, Fratkin JD, Semino-Mora C, Senchak A, Davis MR, \&al. A novel ryanodine receptor gene mutation causing both cores and rods in congenital myopathy. Neurology 2000;55:1689-96.

60. Gonatas NK, Perez MC, Shy GM, Evangelista I. Central "core" disease of skeletal muscle ultrastructural and cytochemical observations in two cases. Am J Pathol 1965;47:503-24.

61. Hayashi K, Miller RG, Brownell AK. Central core disease: Ultrastructure of the sarcoplasmic reticulum and T-tubules. Muscle Nerve 1989;12: 95-102.

62. Oh SJ, Danon MJ. Nonprogressive congenital neuromuscular disease with uniform type 1 fiber. Arch Neurol 1983;40:147-50.

63. Sato I, Wu S, Ibarra MC, Hayashi YK, Fujita H, Tojo M, đal. Congenital neuromuscular disease with uniform type 1 fiber and RYR1 mutation. Neurology 2008;70:114-22
64. Jong YJ, Huang SC, Liu GC, Chiang CH. Mental retardation in congenital nonprogressive myopathy with uniform type 1 fibers. Brain 1991;13:444-6.

65. Caruso JF, Signorile JF, Perry AC, Leblane B, Williams R, Clark M, \& al. The effects of albuterol and isokinetic exercise on the quadriceps muscle group. Med Sci Sports Exerc 1995;27:1471-6.

66. Messina S, Hartley L, Main M, Kinali M, Jungbluth H, Muntoni F, \& al. Pilot trial of salbutamol in central core and multi-minicore diseases. Neuropediatries 2004;35:262-6.

67. Kissel JT, Mendell JR, Griggs RC, McDermott M, Tawil R; The FSHDY group. Open-label clinical trial of albuterol in facioscapulohumeral muscular dystrophy. Neurology 1998;50:1402-6.

Accepted on 30-08-2008

Source of Support: Nil, Conflict of Interest: None declared. 